\title{
Acute Pulmonary Embolism during Puerperium in an HIV Positive Patient Confirmed by Computed Tomography Pulmonary Angiogram
}

\section{Abstract}

Pulmonary embolism is a life-threatening condition associated with high mortality when right ventricular dysfunction is present; anticoagulation and cardiorespiratory supports are the cornerstone of treatment.

Keywords: Pulmonary embolism; High risk; Clinical probability; Computed tomography; Pulmonary angiogram

Received: October 24, 2016; Accepted: January 20, 2017; Published: January 30, 2017

\section{Introduction}

Acute Pulmonary Embolism (PE) is a life-threatening condition which results from occlusion of the pulmonary circulation. The most common situation is when a clot travels from the venous system or right side of the heart, diverting the pulmonary blood flow to the left side of the heart without oxygenation. This creates an intrapulmonary shunt which is the cause of hypoxemia. Acute PE is the most serious clinical presentation of venous thromboembolism [1]. In a study carried out in the United States between 1995 and 2005, the in-hospital fatality rate of patients with primary or secondary diagnosis of acute PE fell from 12.3$8.2 \%$, and the length of hospital stay also decreased from 9.4-8.6 days [2]. Another study showed significant decrease in-hospital fatality rates both in men and women, from 17.6-10.1\% and from 15.6-10.2\%, respectively over the 11 years of study period [3]. Conclusion from those studies support that acute PE is nowadays less lethal than before and cost for hospitalization has declined. The reduction in fatality rate of PE can be associated to better screening tool for $\mathrm{PE}$, so allow to recognize earlier those patients with moderate-high clinical probability of $\mathrm{PE}$, and initiating adequate management.

A case of acute PE in an HIV positive patient is presented. The patient was admitted to Intensive Care Unit in our facility ventilated, stabilized, weaned from the ventilator, and discharged from ICU and later from the hospital.

\section{Case Study}

A 37-year-old female on day 23 after caesarean section with a background of HIV positive on HAART with recent CD4 count 580

\author{
Alexei Ortiz Milan ${ }^{1,2}$, \\ Carlos Medina Merino², \\ Aurelio Rodriguez \\ Fernandez ${ }^{1,2}$ and \\ Megan $\operatorname{Cox}^{1,2}$
}

\section{Faculty of Medicine, University of Botswana, Gaborone, Botswana \\ 2 Intensive Care Unit, Princess Marina Hospital, Gaborone, Botswana}

Corresponding author: Alexei Ortiz Milan

”artizmilan@gmail.com

Faculty of Medicine, University of Botswana, Botswana

Tel: +2673555515

Citation: Milan AO, Merino CM, Fernandez $A R$, et al. Acute Pulmonary Embolism during Puerperium in an HIV Positive Patient Confirmed by Computed Tomography Pulmonary Angiogram. Insights Chest Dis. 2017, 2:1.

$\mathrm{mm}^{3}$, presented to our hospital with Shortness of Breath (SOB) on exertion since delivery. There was no chest pain, no cough and no fever. The patient also had a history of hypertension on treatment (Nifedipine $90 \mathrm{mg}+\mathrm{Hydrochlorothiazide} 25 \mathrm{mg} /$ daily), and treatment for pulmonary TB in 2006. On Physical exam, no remarkable findings were found. Electrocardiogram (Figure 1) was done and it was reported as normal. On admission, she was started on Oxygen and antibiotics (Augmentin+Doxycycline) and Chest- $X$ ray $(C X R)$ was requested.

Six days after admission she was reassessed by physicians still SOB and now complains of chest pain, palpitations, cough, fever and night sweats. Vital signs were normal. CXR from admission was reported with infiltrations in the right lower zones so cotrimoxazole was started as treatment of Pneumocystis Carini Pneumonia (PCP) and screening for Tuberculosis (TB) was initiated. The physician reassessed the following day when the patient had the same symptoms plus left lower limb pain. The notes decribe an unwell looking patient with pallor, pulse rate of 113 beat $/ \mathrm{min}$, saturating $93 \%$ on room air and $98 \%$ on oxygen mask, and physical exam revealed a swollen left calf. The diagnosis of deep venous thrombosis and pulmonary embolism was suspected based on wells score for PE of 7.5 (78.4\% probability of $\mathrm{PE})$, and a left 
lower limb Doppler ultrasound and Pulmonary Artery Computed Tomography (CTPA) were planned while anticoagulation with Enoxaparin $80 \mathrm{mg}$ twice a day subcutaneously was started. The oral anticoagulant warfarin $5 \mathrm{mg}$ was started; daily INR ordered and the physicians requested close monitoring of Blood Pressure $(B P)$, Respiratory Rate (RR), and Pulse Rate (PR). CTPA (Figure 2) reported band-like intraluminal filling defects in the right pulmonary vasculature consistent with pulmonary embolism and there is consolidation of the posterior-basal segment of the right lower lobe.

The patient continued to deteriorate with a tachypnea of $44 / \mathrm{min}$ and oxygen saturations of between $85-90 \%$. Intensive care review was requested due to respiratory distress and desaturating on oxygen to $88-92 \%$. Arterial blood gases showed the following results: $\mathrm{pH} 7.49, \mathrm{PCO} 236, \mathrm{PaO} 2,67, \mathrm{HCO} 326.8$. The patient was transferred to Intensive Care Unit (ICU), intubated and connected to mechanical ventilation on volume assist-control mode and hemodynamic support with noradrenaline was initiated due to severe hypotension. Continuous sedation was given with midazolam and morphine, and anticoagulation continued with Enoxaparin and warfarin. Two days after her admission to ICU was successfully weaned from noradrenaline. Nine days after ICU admission extubation trial is performed which failed, and patient reintubated and sedation is resumed. Patient remained hemodynamically stable throughout her admission in ICU and did not require vasopressors. Four days after reintubation the

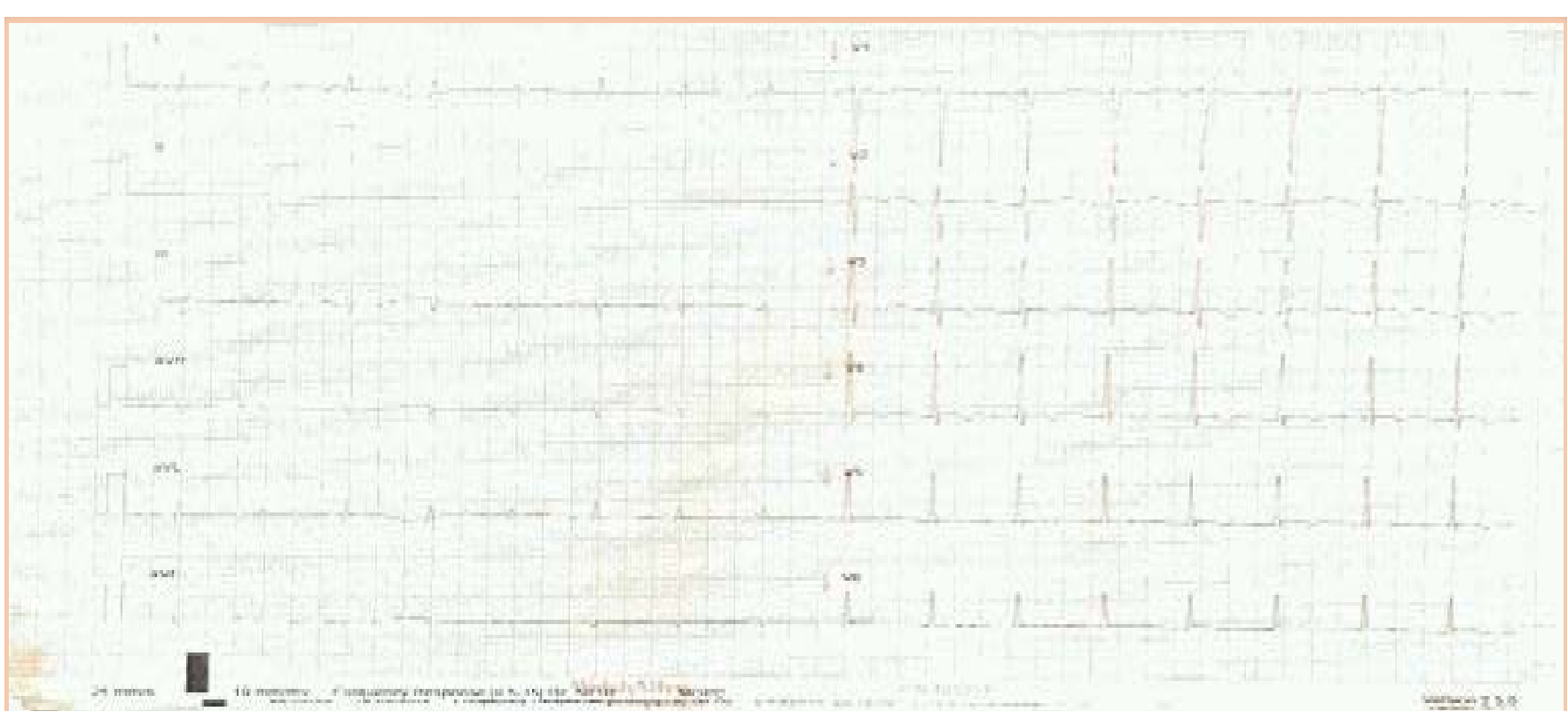

Figure 1 Electrocardiogram showing inverted T from V3 to V5 and flattened in V6.

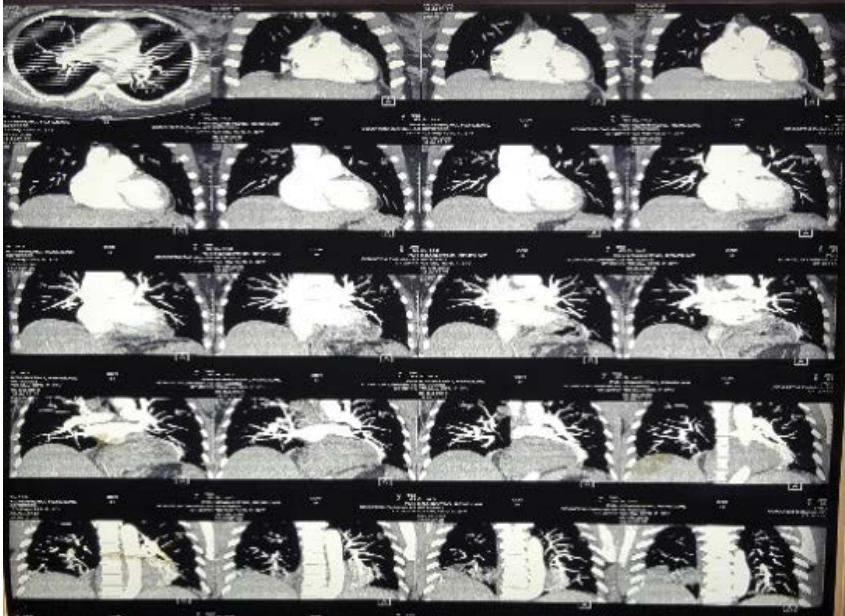

A

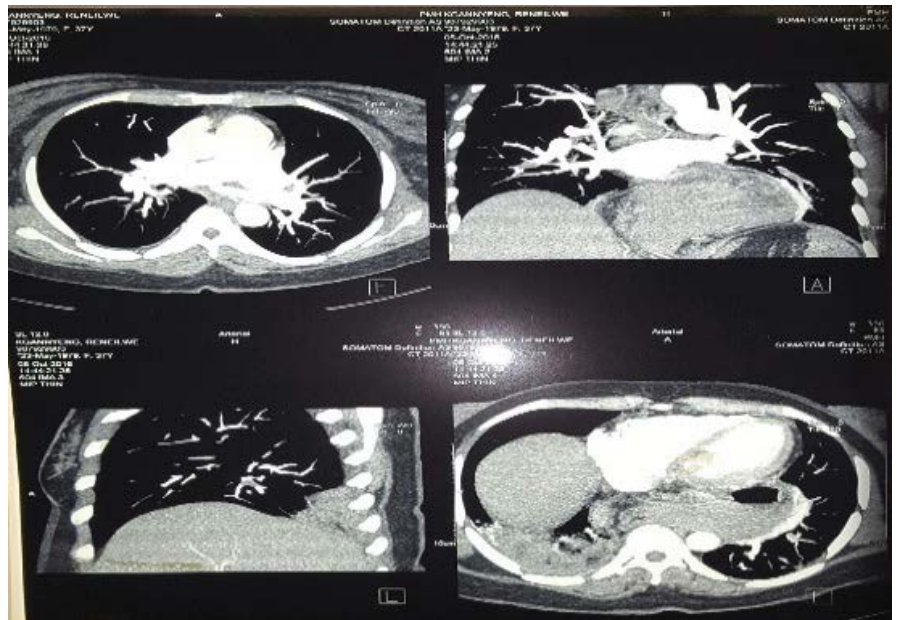

B

Figure 2 Pulmonary artery computed tomography showing intraluminal filling defects in the right pulmonary vasculature (A) and consolidation in the posterior-basal segment of the right lower lobe (B). 
patient was successfully extubated and discharged to medical ward the following day she was discharged home seven days after ICU discharge.

\section{Discussion}

Pulmonary emboli are responsible for $10 \%$ of all deaths in hospital and are a contributory factor in an additional $10 \%$. In contrast, reports of Venous Thromboembolism (VTE) in patients with HIV infection are sparse [4]. VTE is considered to be 'provoked' in the presence of a temporary or reversible risk factor. These factors include surgery, trauma, immobilization, pregnancy, and oral contraceptive use or hormone replacement therapy and can occur within the last 6 weeks to 3 months before diagnosis [5]. PE may also occur in the absence of any known risk factor [1]. If there are no risk factors then the VTE is considered 'unprovoked'. VTE is a major cause of maternal mortality. With the risk highest in the third trimester of pregnancy and over the 6 weeks of the postpartum period. It is up to 60 times higher 3 months after delivery, compared with the risk in non-pregnant women [6].

When the clinical presentation raises the suspicion of PE in an individual patient, it should prompt further objective testing. In most patients, PE is suspected on the basis of dyspnoea, chest pain, pre-syncope or syncope, and/or haemoptysis [1]. Once clinical judgment has raised the suspicion of $\mathrm{PE}$, the assessment of clinical probability is to perform through the well validated Wells score [7]. This score divides the probability of PE in three categories; low-probability (score $\leq 1$ ), moderate-probability (score of 2-6) and high-probability (score $>7$ ), representing 10\%, $30 \%$ and $65 \%$ of probability of $\mathrm{PE}$, respectively. Multi-detector computed tomographic (MDCT) angiography has become the diagnostic tool of choice in patients with suspected $\mathrm{PE}$, and it is the first-line test in patients with high clinical probability in most centres [8]. Magnetic resonance angiography (MRA) has been evaluated as well as diagnostic method in suspected PE but it yields low sensitivity, and there is no availability in our setting. Echocardiography can be used to assess right ventricular function in patient with acute PE; however, there is no individual echocardiographic parameter that provides fast and reliable information on RV size or function [1]. Echo has a negative predictive value of $40-50 \%$, so a negative result cannot exclude PE $[9,10]$. Right ventricular dysfunction in patient with $P E$ has been associated with adverse outcome [11]. RV dysfunction indicates that caution is required with Intravenous fluids expansion as it may worsen the RV function by causing mechanical overstretch, or by reflex mechanisms that depress contractility. A modest fluid challenge $(500 \mathrm{ml})$ may help to increase cardiac index in patients with PE and hypotension [12]. Vasopressors may be used to support the Blood Pressure (BP) when it does not improve with fluid challenge. Norepinephrine appears to improve RV function via a direct positive inotropic effect, while also improving RV coronary perfusion by peripheral vascular alpha-receptor stimulation and the increase in systemic BP. Dobutamine and/ or dopamine may be considered for patients with $\mathrm{PE}$, low cardiac index, and normal BP; however, raising the cardiac index above physiological values may aggravate the ventilation-perfusion mismatch by further redistributing flow from (partly) obstructed to unobstructed vessels. Epinephrine combines the beneficial properties of norepinephrine and dobutamine, without the systemic vasodilatory effects of the latter. It may therefore exert beneficial effects in patients with PE and shock [1]. Anticoagulation, initially parentally with heparin (unfractionated heparin or low molecular weight heparin) over the first 5-10 days overlapping with the initiation of oral vitamin $k$ antagonists (e.g. warfarin) at least for 3 months is recommended and aims to reduce early death and recurrent symptomatic or fatal VTE [13]. The anticoagulant effect of heparin is usually monitored using the aPTT (activated Partial Thromboplastin Time) to achieve an increment of 2-2.5 time above the control aPTT (pre-treatment), and vitamin $\mathrm{k}$ antagonists is monitored through the International Normalized Ratio (INR) to achieve target INR between 2-2.5. Alternatively, one of the new oral anticoagulants (e.g. Dabigatran, endoxaban) can be used. In patients whom presented in shock thrombolysis is recommended.

When mechanical ventilation is required, care should be taken to limit its adverse haemodynamic effects. In particular, the positive intrathoracic pressure induced by mechanical ventilation may reduce venous return and worsen RV failure in patients with massive $\mathrm{PE}$; therefore, positive end-expiratory pressure should be applied with caution. Low tidal volumes (approximately $6 \mathrm{~mL}$ ) kg lean body weight) should be used in an attempt to keep the end-inspiratory plateau pressure less than $30 \mathrm{~cm} \mathrm{H}_{2} \mathrm{O}$ [1].

\section{Conclusion}

Patient with high clinical probability of PE should go straight for Computed Tomography Pulmonary Angiography in order to rule in this pathology. If cardiorespiratory compromise is present, ventilatory and hemodynamic supports should be given along with anticoagulation. In low resource setting when hemodynamic instability is present anticoagulation should be given subcutaneously once hemodynamic stability is achieved together with oral anticoagulant due to difficulties to monitoring coagulation in case of continue infusion of heparin is required.

\section{Acknowledgment}

I would like to recognize the assistance of Mr Joseph, the librarian of the Faculty of Medicine of University of Botswana. 


\section{References}

1 Konstantinides S, Torbicki A, Agnelli G, Danchin N, Fitzmaurice D, et al. (2014) ESC guidelines on the diagnosis and management of acute pulmonary embolism. Eur Heart J.

2 Park B, Messina L, Dargon P, Huang W, Ciocca R, et al. (2009) Recent trends in clinical outcomes and resource utilization for pulmonary embolism in the United States: findings from the nationwide inpatient sample. Chest 136: 983-990.

3 Dentali F, Ageno W, Pomero F, Fenoglio L, Squizzato A, et al. (2016) Time trends and case fatality rate of in-hospital treated pulmonary embolism during 11 years of observation in Northwestern Italy. Thromb Haemost 115: 399-405.

4 Shahmanesh M, Miller RF, Brooks J, Shaw PJ (2000) Inferior vena cava filters for HIV infected patients with pulmonary embolism and contraindications to anticoagulation. Sex Transm Infect 76: 395-397.

5 Kearon C, Akl EA (2014) Duration of anticoagulant therapy for deep vein thrombosis and pulmonary embolism. Blood 123: 1794-1801.

6 Pomp E, Lenselink A, Rosendaal F, Doggen C (2008) Pregnancy, the postpartum period and prothrombotic defects: risk of venous thrombosis in the MEGA study. J Thromb Haemost 6: 632-637.

7 Wells PS, Anderson DR, Rodger M, Stiell I, Dreyer JF, et al. (2001) Excluding pulmonary embolism at the bedside without diagnostic imaging: management of patients with suspected pulmonary embolism presenting to the emergency department by using a simple clinical model and d-dimer. Ann Intern Med 135: 98-107.

8 Murota M, Satoh K, Yamamoto Y, Kobayashi T, Nishiyama Y (2009) Evaluation of subsubsegmental pulmonary arteries of the posterior and anterior segments of the right upper lobe using multidetector row computed tomography with multiplanar reconstruction images. Jpn J Radiol 27: 86-90.

9 Roy PM, Colombet I, Durieux P, Chatellier G, Sors H, et al. (2005) Systematic review and meta-analysis of strategies for the diagnosis of suspected pulmonary embolism. BMJ 331: 259.

10 Grifoni S, Olivotto I, Cecchini P, Pieralli F, Camaiti A, et al. (2000) Shortterm clinical outcome of patients with acute pulmonary embolism, normal blood pressure, and echocardiographic right ventricular dysfunction. Circulation 101: 2817-2822.

11 Kucher N, Rossi E, De Rosa M, Goldhaber SZ (2005) Prognostic role of echocardiography among patients with acute pulmonary embolism and a systolic arterial pressure of $90 \mathrm{~mm} \mathrm{Hg}$ or higher. Arch Intern Med 165: 1777-1781.

12 Mercat A, Diehl JL, Meyer G, Teboul JL, Sors H (1999) Hemodynamic effects of fluid loading in acute massive pulmonary embolism. Intensive Care Med 27: 540-544.

13 Garcia DA, Baglin TP, Weitz JI, Samama MM (2012) Parenteral anticoagulants: antithrombotic therapy and prevention of thrombosis: American College of Chest Physicians evidence-based clinical practice guidelines. Chest 141: e24S-e43S. 